\title{
Isolation of extracellular vesicles from intestinal tissue in a mouse model of intestinal ischemia/reperfusion
} injury

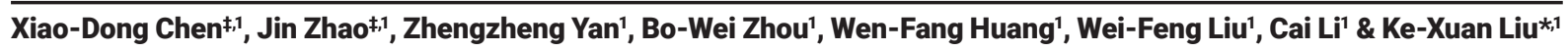

\begin{abstract}
Extracellular vesicles (EVs) are small membranous particles that contribute to intercellular communications. Separating EVs from tissue is still a technical challenge. Here, we present a rigorous method for extracting EVs from intestinal tissue in a mouse intestinal ischemia/reperfusion (I/R) model, and for analyzing their miRNA content. The isolated EVs show a typical cup shape with a size peak of $120-130 \mathrm{~nm}$ in diameter, confirmed by TEM and NTA. They also express EV markers such as CD9, CD63, CD81, Tsg101 and Alix. Real-time qPCR confirmed that these pellets contain miRNAs related to I/R injury. Our study presents a practical way to isolate EVs from intestinal tissue which is suitable for downstream applications such as miRNA analysis, and provides a novel method for investigating the mechanism of intestinal I/R injury.
\end{abstract}

\section{METHOD SUMMARY}

We present a practical way to isolate EVs from intestinal tissue; the method consists of enzymatic digestion, differential centrifugation and density gradient centrifugation. This method is suitable for downstream studies such as miRNA analysis.

\section{KEYWORDS}

extracellular vesicle $\cdot$ intestinal tissue - ischemia reperfusion $\cdot$ miRNA

${ }^{1}$ Department of Anesthesiology, Nanfang Hospital, Southern Medical University, Guangzhou, China; *Author for correspondence: liukexuan705@163.com; ${ }^{\ddagger}$ Authors contributed equally

BioTechniques 68: 257-262 (May 2020) 10.2144/btn-2019-0159
Extracellular vesicles (EVs) are small particles composed of a phospholipid bilayer and enclosed content such as proteins and nucleotides; they participate in intercellular communications by transferring their cargo from donor cells into the target cells. Studying EVs holds significance for understanding disease mechanisms, finding diagnostic markers and developing therapeutic interventions [1]. Isolation of EVs is one of the crucial steps in the current study. Several techniques for EV isolation have been developed, such as ultracentrifugation, polyethylene glycol precipitation, size exclusion chromatography, immunoaffinity capture and microfluidics [2]. There are still no one-size-fits-all solutions for the isolation process, and the choice of methodology must be based on the downstream experiment. Compared with isolating EVs from cell culture media, extracting EVs from tissue is still technically challenging and consensus on tissue EV isolation methodology is lacking.

Intestinal ischemia/reperfusion (I/R) is a grave condition that commonly occurs in various clinical scenarios such as acute mesenteric ischemia, shock and severe infection, and with some surgical procedures including small bowel transplantation, abdominal aneurysm surgery and cardiopulmonary bypass. Intestinal I/R not only causes intestinal barrier damage, but also leads to injuries of remote organs like the lung, liver, heart and brain [3]. Due to the role of EVs as intercellular communication carriers, it is reasonable to hypothesize that gut-derived EVs participate in intestinal I/R injury and act as the link between intestine and remote organs [4]. miRNAs are small noncoding RNA molecules which suppress gene expression by degrading mRNAs or stopping translation, and they have been demonstrated to be related to I/R injuries in the brain, heart and kidney [5]. Our previous studies have identified mucosal miRNA profile changes after intestinal I/R and $m i R$-378-mitigated intestinal injury induced by intestinal I/R [6]. Thus, studying gut-derived EVs and their miRNA cargo is of great interest in understanding the mechanism of intestinal I/R injury and the crosstalk between the intestine and remote organs.

In this study, we present a rigorous method to isolate EVs from small intestine tissue of a mouse intestinal I/R model, and assess the expression of selected miRNAs in these EVs of gut origin after intestinal I/R.

\section{MATERIALS \& METHODS Animals}

Animal experiments were done under the National Institutes of Health guide for the care and use of laboratory animals and in accordance with the regulations for animal welfare and animal experiments of Southern Medical University. Six male C57/BL mice aged 8-10 weeks were used, three in the I/R group and three in the control group. All the animals were bought from the laboratory animal center of Southern Medical University, and kept in a specific pathogenfree environment.

\section{Reagents}

HBSS buffer (Lot:2042327) was bought from Gibco (NY, USA). Type 1 collagenase (lot: 48S18874) was bought from Worthington Biochemical Corporation (NJ, USA). OptiPrep $^{\text {TM }}$ Density Gradient Medium (lot: BCCB2082) was bought from Sigma (MO, USA). Trehalose (lot: S25319) was bought from Shanghai Yuanye Biotechnology Company (Shanghai, China). Complete $^{\mathrm{TM}}$ protease inhibitor cocktail (lot: 4693116001) was bought from Roche (Basel, Switzerland). 10x cell lysis buffer (\#9803) was bought from Cell Signaling Technology (MA, USA). Bicinchoninic acid 
(BCA) protein assay kit (lot: TH269580) was bought from Thermo Scientific (MA, USA). Mouse endotoxin ELISA kit (ml002005) was bought from Shanghai Mlbio Company (Shanghai, China). miRNeasy Serum/Plasma Kit (lot: 217184) was bought from Qiagen (Duesseldorf, Germany). Mir-X miRNA FirstStrand Synthesis Kit (638315) and TB Green ${ }^{\circledR}$ Premix Ex Taq ${ }^{\text {TM }}$ (RR420L) were bought from Takara (Tokyo, Japan).

\section{Surgical procedure}

General anesthesia was induced by inhalation of $4 \%$ isoflurane and then maintained at $1.5 \%$ isoflurane throughout the procedure. A warming pad was placed under the mouse to prevent hypothermia. Abdominal skin was shaved and sterilized. A 1-cm midline laparotomy was made, and intestine was gently dragged out of the abdominal cavity using wet cotton swabs. In the I/R group, the superior mesenteric artery was identified and occluded by a noninvasive vessel microclip for $30 \mathrm{~min}$; the clip was then removed and the intestine replaced in the peritoneal cavity. In the control group, the intestine was exteriorized and returned into the abdomen without clipping the superior mesenteric artery. The wound was closed by interrupted suture. The mouse was kept under isoflurane anesthesia until tissue harvest. After $2 \mathrm{~h}$ of reperfusion, the mouse was killed by transcardial perfusion of ice-cold saline. The abdominal incision was reopened, the whole length of small intestine was harvested, and the mesentery was removed. The luminal content was flushed out by injecting ice-cold saline with a $20-\mathrm{ml}$ syringe.

\section{Histology}

A $0.5-\mathrm{cm}$-long section of the intestine, $5 \mathrm{~cm}$ proximal to the ileocecal valve, was cut off and then fixed in $4 \%$ paraformaldehyde overnight. The sections were then embedded in paraffin wax and proceeded to routine protocol as described previously [7]; three histological sections from each sample were stained with hematoxylin and eosin. The slides were observed and photographed with an Olympus BX51 microscope.

\section{EV isolation}

The fresh intestine was placed on ice, opened longitudinally and then cut into segments of less than $0.5 \mathrm{~cm}$ in length. The tissue pieces were then soaked in HBSS buffer with type I collagenase at a concentration of 300 units $/ \mathrm{ml}$, and rotated slowly for $30 \mathrm{~min}$ at $37^{\circ} \mathrm{C}$. The enzymatic digestion was stopped by adding an equal volume of phosphate-buffered saline (PBS) with $2 \times$ protease inhibitor cocktail. The lysate was then passed through a $70-\mu \mathrm{m}$ strainer. The filtrate was centrifuged successively at $1000 \times \mathrm{g}$ for $10 \mathrm{~min}, 2000 \times \mathrm{g}$ for $20 \times \mathrm{min}$, $5000 \times \mathrm{g}$ for $30 \mathrm{~min}$ and $15,000 \times \mathrm{g}$ for $1 \mathrm{~h}$ at $4^{\circ} \mathrm{C}$. The supernatant was then filtered through a $0.22-\mu \mathrm{m}$ Millex PVDF filter and ultracentrifuged at $120,000 \times \mathrm{g}$ for $130 \mathrm{~min}$ in a Beckman SW32Ti rotor at $4^{\circ} \mathrm{C}$. After ultracentrifugation, the supernatant was poured off and the pellet resuspended and washed in $25 \mathrm{mM}$ trehalose/PBS, then ultracentrifuged as described above. The density gradient was set up by carefully laying $4 \mathrm{ml}$ $40 \%, 3 \mathrm{ml} 20 \%$ and $3 \mathrm{ml} 5 \%$ Optiprep iodixanol solutions (prepared according to the manufacturer's instructions) in a bottom-up manner. The pellet, in $1 \mathrm{ml}$ trehalose/PBS, was loaded carefully on top of the density gradient, and then centrifuged at $288,000 \times g$ in the Beckman SW41Ti rotor for $5 \mathrm{~h}$ at $4^{\circ} \mathrm{C}$. A parallel density gradient without pellet samples was also spun in the same rotor. After centrifugation, the density medium was carefully pipetted from the surface and divided into ten fractions from top to bottom, in order. Each fraction was transferred to a new tube and centrifuged at $100,000 \times g$ for $70 \mathrm{~min}$ in the Beckman Type 70.1Ti rotor. Each pellet was resuspended in $200 \mu \mathrm{l}$ $25 \mathrm{mM}$ trehalose/PBS and stored at $-80^{\circ} \mathrm{C}$.

\section{Nanoparticle tracking analysis}

The optimal dilution ratio was determined prior to measurement. Double-distilled water was degassed by ultrasound. The chamber of the ZetaView instrument was flushed and filled with particle-free water using a $20-\mathrm{ml}$ syringe. After camera adjustment, the sample was injected into the chamber with a 1-ml syringe, avoiding bubbles. Particle size and concentration were recorded, and each sample was measured in triplicate.

\section{Transmission electron microscopy}

The optimal concentration of EVs for electron microscopic observation was determined and the EV/PBS suspension diluted accordingly with double-distilled water. A drop of diluted EV was placed on a clean plate, mixed thoroughly with an equal amount of phosphotungstic acid by pipetting, and stained for $10 \mathrm{~min}$. The sample was then loaded on a copper grid and air dried. The copper grids with samples were then mounted for electron microscopic imaging under $80 \mathrm{kV}$.

\section{Protein extraction}

The $2 \times$ cell lysis buffer, supplemented with protease inhibitor cocktail, was added to an equal volume of resuspended EVs, mixed thoroughly, sonicated at $30 \%$ AMP by a Qsonica Q800R3 sonicator for $5 \mathrm{~min}$ and centrifuged at $13,000 \mathrm{~g}$ for $15 \mathrm{~min}$. The supernatant was collected and transferred to a new Eppendorf tube. The protein concentration was determined by a BCA assay kit according to the manufacturer's instructions.

\section{Western blot}

The protein lysate was mixed with $5 \times$ loading buffer and boiled at $95^{\circ} \mathrm{C}$ for $5 \mathrm{~min}$, then loaded into the 12 or $6 \%$ SDS-PAGE gel. After electrophoresis, the separated proteins were transferred onto the 0.45 or $0.22 \mu \mathrm{m}$ PVDF membrane. The membrane was blocked with milk and then incubated with the following primary antibodies at $4^{\circ} \mathrm{C}$ overnight: rabbit anti-CD9 (1:1000, ab92726, Abcam, MA, USA), anti-CD63 (1:2000, ab217345, Abcam), anti-CD81 (1:1000, \#10037, Cell Signaling Technology), anti-Tsg101 (1:1000, ab125011, Abcam), anti-Alix (1:500, 12422-1-AP, Proteintech, IL, USA), anti- $\beta$-actin (1:3000, AP0060, Bioworld, Nanjing, China), anti-ompA (1:1000, orb6940, Biorbyt, Cambridge, UK), anti-ompC (1:1000, orb422682, Biorbyt) and anti-CD45 (1:500, 20103-1-AP, Proteintech). The membrane was then washed three-times and incubated with secondary antibody. Proteins were detected by a Tanon 5200 Chemiluminescent Imaging System (Shanghai, China) with a Millipore Immobilon Western Chemiluminescent HRP kit (WBKLS0500, Millipore, MA, USA) according to the manufacturer's instructions.

\section{miRNA extraction}

Total RNAs were extracted from EVs with miRNeasy serum/plasma Kit according to the manufacturer's instructions: Briefly, five volumes of QIAzol lysis reagent were added to $200 \mu \mathrm{l} \mathrm{EV} \mathrm{suspensions.} \mathrm{Synthetic}$ 


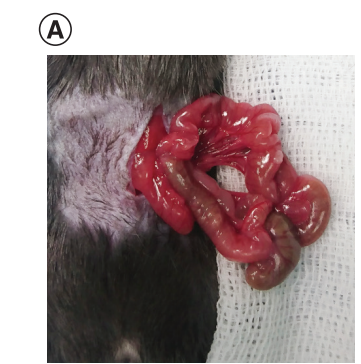

(C)

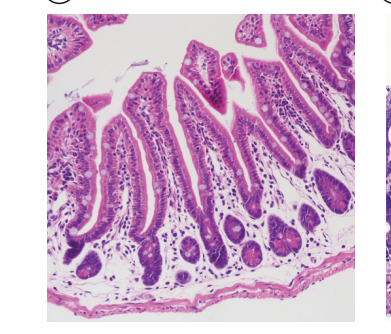

(B)

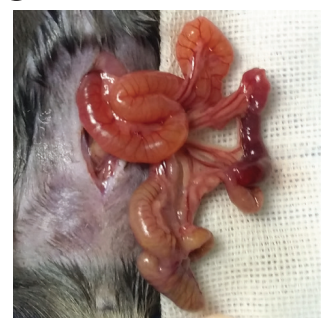

(D)

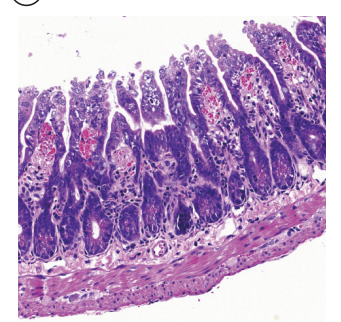

(E)

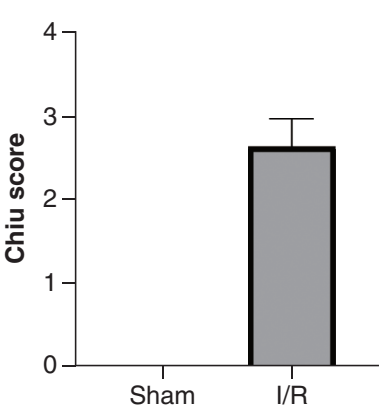

Figure 1. Morphological changes after intestinal ischemia and reperfusion. The superior mesenteric artery was clipped for $30 \mathrm{~min}$ and allowed to reperfuse for $2 \mathrm{~h}$. (A \& C) Macroscopic and microscopic appearances of intestine in the control group. (B \& D) Macroscopic and microscopic appearances of intestine in the I/R group. (E) Scoring of histological injury according to a Chiu's grading system, $n=3$ in each group.

I/R: Ischemia/reperfusion.

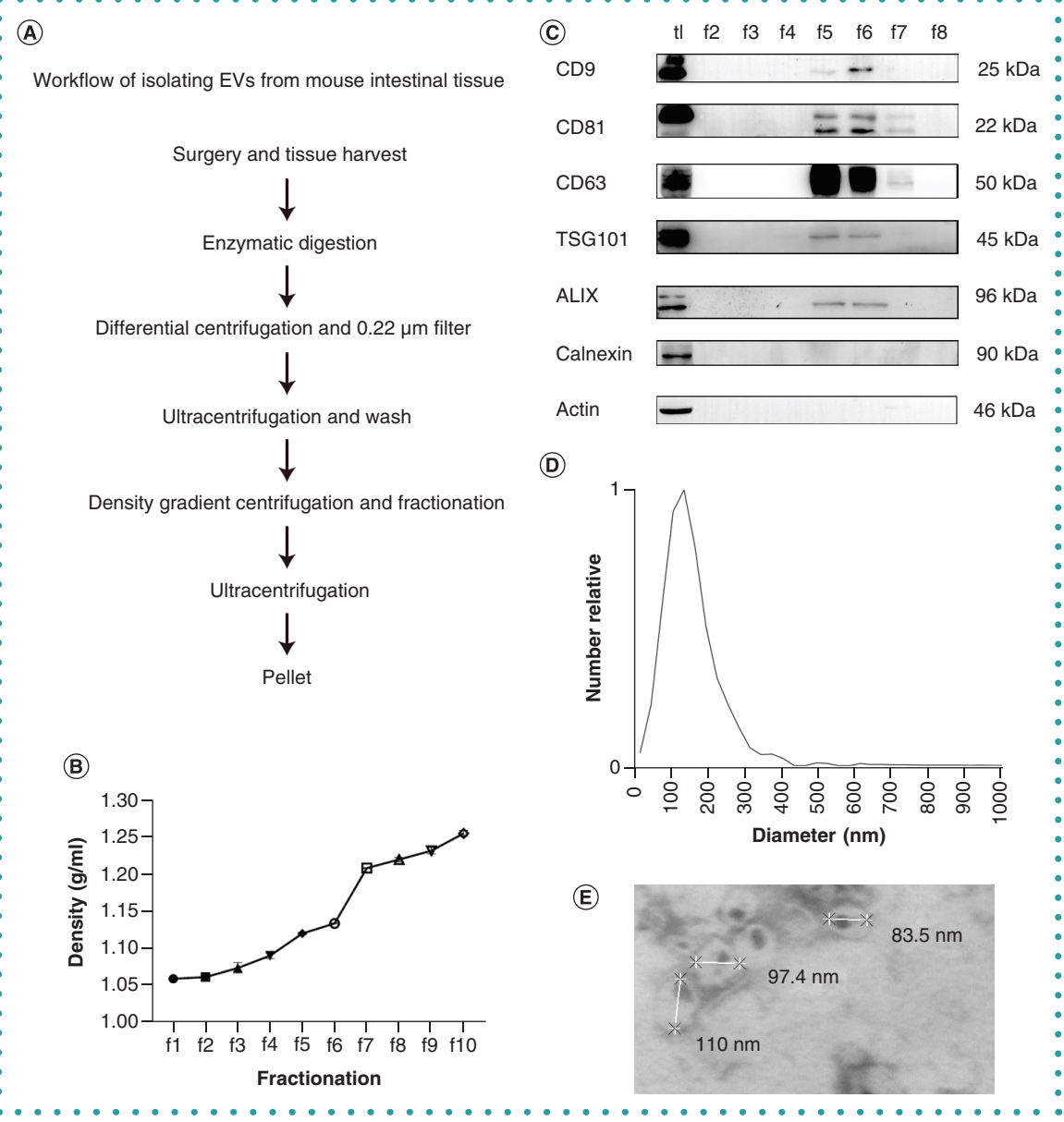

Figure 2. Isolation and characterization of extracellular vesicles from ischemia/reperfusion intestinal tissue. (A) Flowchart of EV isolation procedures. (B) The initial density gradient of 40,20 and $5 \%$ formed a more linear distribution after centrifugation at $288,000 \times \mathrm{g}$ for $5 \mathrm{~h}$ in a SW41Ti rotor; the supernatant was fractionated from the top, 1-ml volume for each fraction. (C) Western blot of intestinal tissue lysate (TL), and second fraction (f2) to eighth fraction (f8); EV markers CD9, CD63, CD81, Alix, and Tsg101 are positive in $\mathrm{f} 5$ and $\mathrm{f} 6$. (D) $\mathrm{f} 5$ and $\mathrm{f} 6$ were pooled for nanoparticle tracking analysis; the particle diameter is approximately $120-130 \mathrm{~nm}$. (E) Transmission electron microscopy of pooled $\mathrm{f} 5$ and $\mathrm{f} 6$ shows typical cup-shaped EVs with diameters ranging from 80 to $110 \mathrm{~nm}$. EV: Extracellular vesicle.
Cel-miR-39 was introduced into the lysate as a spike-in control before adding chloroform. After the addition of chloroform, the lysate was separated into aqueous and organic phases by centrifugation. RNAs in the aqueous phase were extracted, and ethanol added. The sample was transferred to the RNeasy MinElute spin column, to remove phenol and other contaminants; RNAs were then eluted in a small volume of RNase-free water.

\section{miRNA reverse transcription \& qPCR}

A Clontech miRNA First-Strand Synthesis kit was used for miRNA reverse transcription according to the manufacturer's manual. Real-time PCR was performed with a Takara TB Green premix on Applied Biosystems QuantStudio $^{\text {TM }} 5$ Real-Time PCR System. Primer sequences were as follows: cel-miR39: CCTCACCGGGTGTAAATCAGCTTG, let-7d: GCTATACGACCTGCTGCCTTTCT, miR-21a: CGCCGTAGCTTATCAGACTGATGTTGA, miR-34a: GTGGCAGTGTCTTAGCTGGTTGT, miR-122: CCGTGGAGTGTGACAATGGTGTTTG, miR-155: CGCCGTTAATGCTAATTGTGATAGGGG and miR-210: CTGTGCGTGTGACAGCGG, miR-221: CCAGCTACATTGTCTGCTGGGTTTC. For the relative quantification of miRNA expression, the delta cycle threshold (CT) method was applied, with the miR-39 spike-in control used as the reference gene.

\section{Statistical analysis}

The graphs are presented as \pm SEM. The Graph Pad Prism, 8th version (GraphPad 


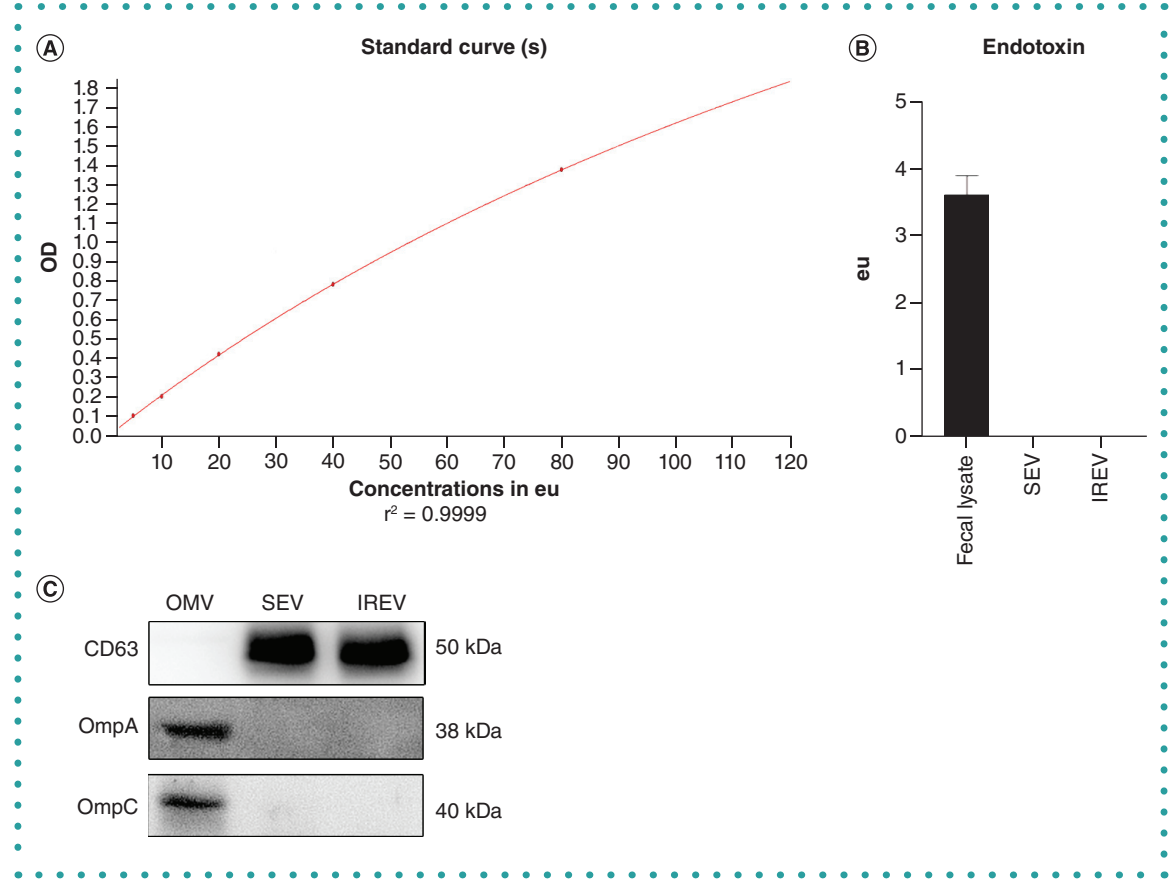

Figure 3. Examination of bacterial contamination of isolated extracellular vesicles. Endotoxin assay of EV sample with an ELISA kit. (A) Standard curve. (B) Endotoxin was not detectable in EVs isolated from SEV or IREV; mouse cecum fecal lysate was used as a positive control. (C) OmpA and ompC were negative in EV lysate determined by western blot; OMVs isolated from human feces were used as positive control.

EV: Extracellular vesicle; IREV: EVs isolated from ischemia/reperfusion group; OD: Optical density; SEV: EVs isolated from control group.

Software, CA, USA), was used for statistical analysis. Student's $t$-test was adopted to compare results between two groups as appropriate and statistical significance was indicated as: ${ }^{*} \mathrm{p} \leq 0.05$.

\section{RESULTS \& DISCUSSION}

\section{Morphological changes of intestine}

\section{after ischemia \& reperfusion}

The intestinal I/R model was built by clipping the superior mesenteric artery for $30 \mathrm{~min}$, followed by reperfusion for $2 \mathrm{~h}$. Compared with the control group (Figure $1 \mathrm{~A} \& \mathrm{C}$ ), a segmental lesion of darker color and diffused dilatation of the intestine can be seen macroscopically in the I/R group (Figure 1B). Histological changes of $\mathrm{I} / \mathrm{R}$ intestine including bleb formation at the villi tips, epithelial cell detachment, villus tip denudating and intravillus bleeding was observed microscopically (Figure 1D). A Chiu's scoring of 2 to 3 grade was assigned (Figure 1E) [8].

\section{Intestinal tissue EV isolation \& charac- terization}

To minimize possible contamination, the luminal content of the intestine was flushed out using cold PBS and blood was expelled from the intestinal tissue by transcardial perfusion from the left ventricle with ice-cold normal saline. After enzymatic digestion, protease inhibitor cocktail was added into the lysate to protect the EV surface proteins. Any large floating particles were cleared using $70-\mu \mathrm{m}$ mesh filters. Cells, cell debris and other non-EV particles were pelleted and removed by differential centrifugation and a 0.22- $\mu \mathrm{m}$ filter (Figure 2A). At this stage, a crude EV precipitate was visible at the bottom of the tube.

After ultracentrifugation for $5 \mathrm{~h}$, the initial density gradient of $40 \%, 20 \%$ and $5 \%$ formed a more linear distribution. Removing $1 \mathrm{ml}$ of each fraction from the top, it was found that the densities of the fifth and sixth fractions were well within the range of exosomes $(1.12-1.19 \mathrm{mg} / \mathrm{ml}$ ) (Figure 2B) [1]. Western blotting showed that exosome markers CD9, CD63, Tsg101, Alix and CD83 were present in these two fractions, whereas calnexin, an indicator of intracellular component contamination, was not found (Figure 2C). Then the fifth and sixth fractions were pooled together and subjected to NTA to determine the particle number and size distribution; the results showed that the particle size was approximately $120-130 \mathrm{~nm}$ in diameter and the concentration 2-4 $\times 10^{11}$ particles per $\mathrm{ml}$ (Figure 2D). The EV morphology in the pooled fifth and sixth fractions was also examined under TEM. Typical cup-shaped structures were observed and the diameter was around $100 \mathrm{~nm}$ (Figure 2E).

To assess possible bacterial contamination, a commercial endotoxin ELISA kit was used to test the endotoxin level of isolated EVs; fecal lysate from mouse cecum was used as a positive control. Endotoxin was not detectable in EV samples (Figure 3A \& B). OmpA and ompC, two markers of bacteria-derived outer membrane vesicles (OMVs), were also negative in EV samples determined by western blot. OMVs isolated from human stools were used as a positive control.

\section{RNA content of EV}

Total RNAs were extracted from the purified EVs using a commercial column-based kit with $100 \mu \mathrm{g}$ of EV from each sample in $200 \mu \mathrm{l}$ PBS. The quality and quantity of total RNAs was determined using NanoDrop and the Agilent bioanalyzer. The A260/280 ratio was around 2, and RNA concentrations were 30 to $50 \mathrm{ng} / \mu \mathrm{l}$. The bioanalyzer showed that the RNAs mainly consisted of small RNAs (Figure 4A). Selected miRNAs (including let7d, miR-21a, miR-34a, miR-122, miR-155, miR-2 10 , and miR-221) have been reported to be involved in the pathophysiological processes of I/R injuries [5,9]. RT-qPCR was utilized to assess the differential expressions of these miRNAs between EVs from the control and I/R groups. The CT value of miR-21a was around 20 , indicating its relatively high expression in intestinal tissue-derived EVs (Figure 4B). When normalized to spike-in control, the expression level of miR-221 in the I/R EVs was more than threefold higher than in the control EVs. Expression levels of let-7d, miR-21a, miR-34a, miR-122, miR-155, and miR-210 in I/R EVs showed an increasing trend but were not statistically significant (Figure 4C).

In this study, we successfully separated EVs from intestinal tissue of a mouse intestinal I/R model. Unlike traditional EV separation strategies, we used in vivo tissue as starting material instead of cell culture media or biological fluids, and employed gentle collagenase digestion to free the extracellular space with minimized disruption of 
cells. We stopped the enzymatic activity by adding protease inhibitors, protecting the surface markers of dissociative EVs from degradation. We utilized more osmosisfriendly iodixanol, instead of sucrose, as the density gradient centrifugation medium for EV purification. We resuspended the EV pellet with trehalose/PBS to reduce EV aggregation.

Ultracentrifugation has become the gold standard EV isolation method for cell culture media and biological fluids $[10,11]$. Our previous studies have demonstrated that apoptosis and necroptosis are the major form of cell death in intestinal I/R [12]. Since the release of apoptotic bodies and other small vesicles from these two processes will contaminate the EV pellet after ultracentrifugation, further purification is necessary. Density gradient centrifugation can be utilized to separate impurities such as lipid, protein and nucleotide aggregates from EVs due to their buoyant density [13]. It can also efficiently remove apoptotic bodies and microvesicles larger than $200 \mathrm{~nm}$ in diameter from small EV (exosome) fractions [14]. One problem of ultracentrifugation is EV pellet aggregation and adhesion. Trehalose is nontoxic and widely used as a food additive and drug preservative; recent studies have proved its protective role in stabilizing proteins and RNA in EVs isolated from cell culture medium or biofluids, and it also prevents EV aggregation during isolation and protects them from freeze-thaw damage [15,16].

Hypoxia/reoxygenation (H/R) of epithelial cell cultures is a common model for studying intestinal I/R injury in vitro; thus, H/R cell culture-conditioned medium is a common source for the isolation of I/R related EVs. For this purpose, primary cell cultures or immortalized cell lines are the two major choices. In order to yield enough EVs, multiple cell cultures and media collection and concentration are necessary. Procedures for isolating primary cells from intestinal tissue are often sophisticated. Cell lines are genetically modified and can be structurally or functionally changed compared with their in vivo counterparts. Cultured cells also lack the local environment for interaction with other cell types. EV cargos differ when the physiological state and local milieu of their donor cells are changed; thus EVs from H/R-conditioned media may not
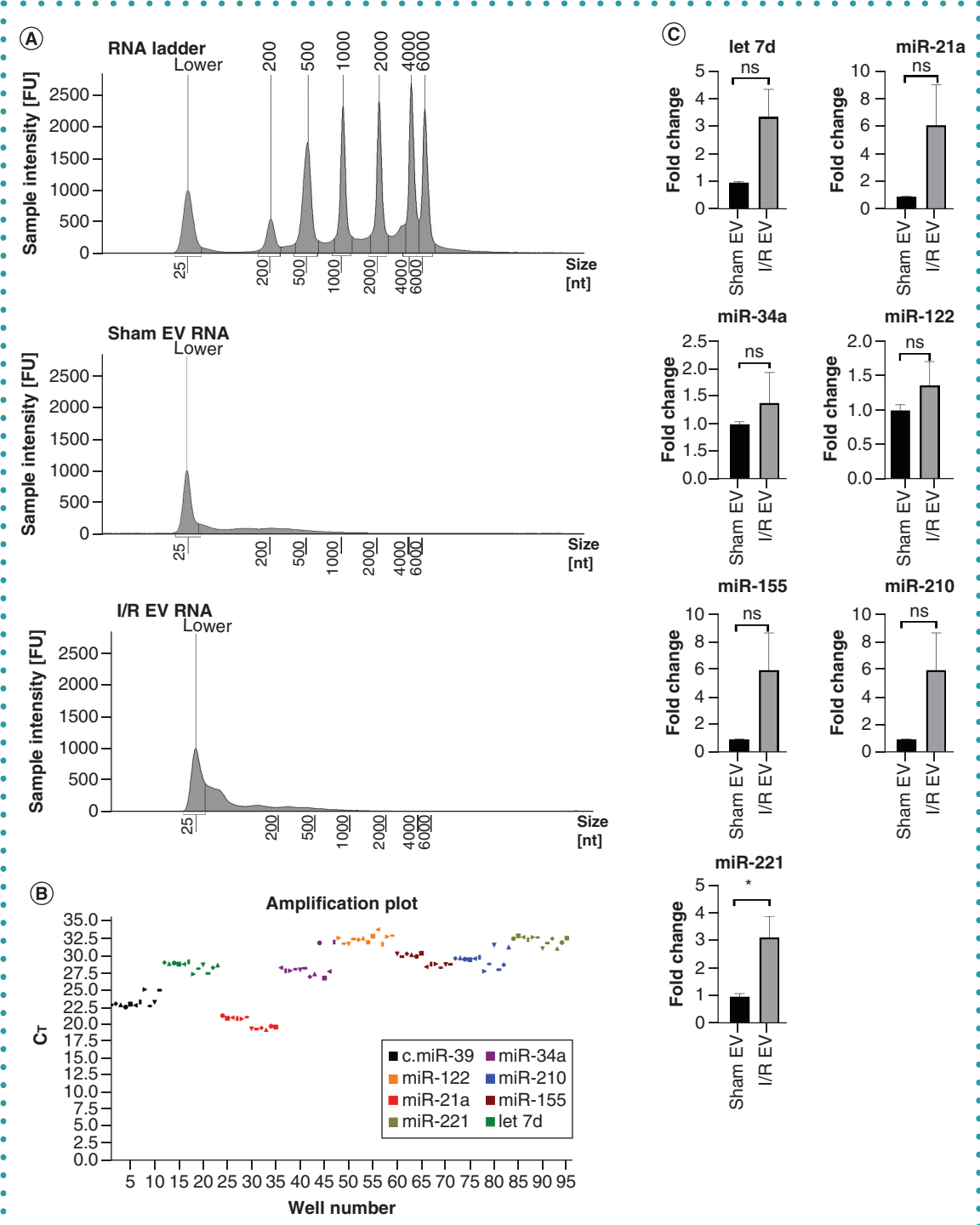

Figure 4. Analysis of RNA content of intestinal extracellular vesicles. (A) Total RNA was extracted from intestinal EVs of control and I/R mice and the nucleotide length was measured by Agilent Bioanalyzer 2200. (B) A representative amplification plot of qPCR from three independent experiments; each target was measured in two wells as technical duplicates, and the CT values of each well are shown. (C) Relative quantification of selected miRNAs ( $n=3$ in each group). Student's $t$ test was used to compare results between the two groups; statistical significance is indicated as ${ }^{*} \mathrm{p} \leq 0.05$.

$\mathrm{C}_{\mathrm{T}}$ : Cycle threshold; EV: Extracellular vesicle; FU: Fluorescence; I/R: Ischemia/reperfusion.

mimic the reality of intestinal I/R. For these reasons, to study the function of EVs in the mechanism of intestinal I/R injury and intestinal I/R-induced remote organ injuries it is advisable to use tissue-origin EVs which might better reflect the real situation.

Extracting EVs from tissue has been reported, using methods based on commercial polyethylene glycol kits, ultracentrifugation or sucrose density gradient centrifugation [17,18]. However, contaminants such as protein aggregate and biopolymer molecules tend to be coprecipitated and interfere with downstream experiments, including proteomic analysis and RNA assays [14]. The osmolality of the iodixanol gradient solution is in the range 295-310 mOsm, which is complementary to the membranous structures of EVs, making it a good candidate for density gradient medium in EV purification [19].

The role of EVs in heart, brain and kidney ischemia reperfusion injuries has been explored. By transmitting cargo 
to the recipient cells, EVs exert an aggravating or protective effect in I/R injuries in these organs. miR-21a has been reported to target PTEN, increase fibroblast metalloprotease-2 expression and contribute to cardiac dysfunction after heart ischemia [5]. However, a recent study also demonstrated its protective role in myocardial infarction by limiting cell apoptosis [20]. miR-122 and $m i R-210$ are involved in transient ischemic attack or stroke [21,22], and miR-122 is also related to warm ischemia in liver transplantation [23]. miR-155 is a potent contributor to IR injuries of the brain, heart and liver [24,25]. miR-221 is cardioprotective and has antiapoptotic and antiautophagic functions in myocardial infarction; it also exerts a protective role in cerebral ischemic stroke by promoting angiogenesis [26-28]. Exploration of the functions of miRNAs in gut-derived EVs in the context of intestinal $\mathrm{I} / \mathrm{R}$ is therefore warranted.

\section{FUTURE PERSPECTIVE}

In this study, we present a practical way to isolate EVs from intestinal tissue with relatively high purity and productivity, suitable for downstream studies such as miRNA analysis. Our study also provides a novel method to investigate the mechanism of intestinal I/R injury. In the future, we hope to divide the subpopulations of these EVs according to their parent cells and to clarify the functions of individual subpopulations. The in vivo tracking of gut tissue-derived EVs through humoral circulation is also necessary to investigate their roles in crosstalk between the intestine and remote organs during intestinal I/R.

\section{AUTHOR CONTRIBUTIONS}

X-D Chen: study design, EV extraction, data collection and drafting of the paper; J Zhao: EV marker analysis and data collection; Z Yan: animal experiment; B-W Zhou: analysis of data; W-F Huang: animal experiment; W-F Liu: analysis and interpretation of data; C Li: revising the article critically for important intellectual content; and K-X Liu: study design and revision of the whole paper and supervising the entire study. All authors approved the final edition of the manuscript.

\section{ACKNOWLEDGMENTS}

The authors thank Guangzhou RIBOBIO Company for their help with microRNA bioanalysis. We also thank $\mathrm{P}$ Chen from Southern Medical University for giving us OMV samples.

\section{FINANCIAL \& COMPETING INTERESTS DISCLOSURE}

This work was supported by grants from National Natural Science Foundation, Beijing, China (81671955 to K-X Liu), Key Program of National Natural Science Foundation, Beijing, China (81730058 to K-X Liu) and PhD Start-up Fund of Natural Science Foundation of Guangdong Province (2017A030310118 to Z Yan). The authors have no other relevant affiliations or financial involvement with any organization or entity with a financial interest in or financial conflict with the subject matter or materials discussed in the manuscript apart from those disclosed.

No writing assistance was utilized in the production of this manuscript.

\section{ETHICAL CONDUCT OF RESEARCH}

The authors state that they have obtained appropriate institutional review board approval or have followed the principles outlined in the Declaration of Helsinki for all human or animal experimental investigations.

\section{OPEN ACCESS}

This work is licensed under the AttributionNonCommercial-NoDerivatives 4.0 Unported License. To view a copy of this license, visit http://creativecommons.org/licenses/ by-nc-nd/4.0/

\section{REFERENCES}

Papers of special note have been highlighted as: $\cdot$ of considerable interest

1. Mathieu M, Martin-Jaular L, Lavieu G, Théry C. Specificities of secretion and uptake of exosomes and other extracellular vesicles for cell-to-cell communication. Nat. Cell Biol. 21(1), 9-17 (2019).

2. Mateescu B, Kowal EJK, van Balkom BWM et al. Obstacles and opportunities in the functional analysis of extracellular vesicle RNA - an ISEV position paper. Extracell. Vesicles 6(1), 1286095 (2017).

-. Addresses the general requirement for extracellular vesicle (EV) miRNA studies and the advantages of EV isolation methods on downstream EV RNA research.

3. Zhou J, Huang WQ, Li C et al. Intestinal ischemia/reperfusion enhances microglial activation and induces cerebral injury and memory dysfunction in rats. Crit. Care Med. 40(8), 2438-2448 (2012).

4. Kojima M, Costantini TW, Eliceiri BP, Chan TW, Baird A Coimbra R. Gut epithelial cell-derived exosomes trigger post-trauma immune dysfunction. J. Trauma Acute Care Surg. 84(2), 257-264 (2018)

5. Weiss JB, Eisenhardt SU, Stark GB, Bode C, Moser M, Grundmann S. MicroRNAs in ischemia-reperfusion injury. Am. J. Cardiovasc. Dis. 2(3), 237-247 (2012).

6. Li Y, Wen S, Yao $X$ et al. MicroRNA-378 protects against intestinal ischemia/reperfusion injury via a mechanism involving the inhibition of intestinal mucosal cell apoptosis. Cell Death Dis. 8(10), e3127 (2017).

7. Zhang XY, Liu ZM, Zhang HF et al. TGF-beta1 improves mucosal IgA dysfunction and dysbiosis following intestinal ischaemia-reperfusion in mice. J. Cell. Mol. Med. 20(6), 1014-1023 (2016).

8. Chiu CJ, McArdle AH, Brown R, Scott HJ, Gurd FN Intestinal mucosal lesion in low-flow states. I. A morphological, hemodynamic, and metabolic reappraisal. Arch. Surg. 101(4), 478-483 (1970).

9. Lin W-B, Liang $M-Y$, Chen G-X et al. MicroRNA profiling of the intestine during hypothermic circulatory arrest in swine. World J. Gastroenterol. 21(7), 2183-2190 (2015).

10. Théry $C$, Witwer KW, Aikawa E et al. Minimal information for studies of extracellular vesicles 2018 (MISEV2018): a position statement of the International Society for Extracellular Vesicles and update of the MISEV2014 guidelines. J. Extracell. Vesicles 7(1), 1535750 (2018).

.- States the need for, and challenge of, isolating tissue EVs.

11. Langevin SM, Kuhnell D, Orr-Asman MA et al. Balancing yield, purity and practicality: a modified differential
ultracentrifugation protocol for efficient isolation of small extracellular vesicles from human serum. RNA Biol. 16(1), 5-12 (2019).

12. Wen $S$, Ling $Y$, Yang $W$ et al. Necroptosis is a key mediator of enterocytes loss in intestinal ischaemia/reperfusion injury. J. Cell. Mol. Med. 21(3), 432-443 (2017).

13. Lobb RJ, Becker M, Wen Wen S et al. Optimized exosome isolation protocol for cell culture supernatant and human plasma. J. Extracell. Vesicles 4(1), 27031 (2015).

14. Konoshenko MY, Lekchnov EA, Vlassov AV, Laktionov PP. Isolation of extracellular vesicles: general methodologies and latest trends. Biomed Res. Int. 2018, 27 (2018).

15. Charoenviriyakul C, Takahashi Y, Nishikawa M, Takakura Y. Preservation of exosomes at room temperature using lyophilization. Int. J. Pharm. 553(1), 1-7 (2018).

16. Neo SH, Chung KY, Quek JM, Too H-P. Trehalose significantly enhances the recovery of serum and serum exosomal miRNA from a paper-based matrix. $\mathrm{Sci}$. Rep. 7(1), 16686 (2017).

17. Loyer $X$, Zlatanova I, Devue $C$ et al. Intra-cardiac release of extracellular vesicles shapes inflammation following myocardial infarction. Circ. Res. 123(1), 100-106 (2018).

- A novel method of isolating EVs from myocardial tissue.

18. Vella LJ, Scicluna BJ, Cheng $L$ et al. A rigorous method to enrich for exosomes from brain tissue. J. Extracell. Vesicles 6(1), 1348885 (2017).

- A novel method of isolating EVs from brain tissue.

19. Iwai K, Minamisawa T, Suga K, Yajima Y, Shiba K. Isolation of human salivary extracellular vesicles by iodixanol density gradient ultracentrifugation and their characterizations. J. Extracell. Vesicles 5(1), 30829 (2016).

20. Luther KM, Haar L, McGuinness $M$ et al. Exosomal miR-21a-5p mediates cardioprotection by mesenchyma stem cells. J. Mol. Cell. Cardiol. 119, 125-137 (2018).

21. Ghoreishy A, Khosravi A, Ghaemmaghami A. Exosomal microRNA and stroke: a review. J. Cell. Biochem. 120(10), 16352-16361 (2019).

22. Li D-B, Liu J-L, Wang W et al. Plasma exosomal miRNA122-5p and miR-300-3p as potential markers for transient ischaemic attack in rats. Front. Aging Neurosci. 10 24 (2018).

23. Farid WRR, Pan Q, van der Meer AJP et al. Hepatocyte-derived microRNAs as serum biomarkers of hepatic injury and rejection after liver transplantation. Liver Transpl. 18(3), 290-297 (2012).

24. Tan L, Jiang W, Lu A, Cai H, Kong L. miR-155 aggravates liver ischemia/reperfusion injury by suppressing SOCS in mice. Transplant. Proc. 50(10), 3831-3839 (2018).

25. Li JJ, Wang B, Kodali MC et al. In vivo evidence for the contribution of peripheral circulating inflammatory exosomes to neuroinflammation. J. Neuroinflammation 15(1), 8 (2018).

26. Zhou Y, Richards AM, Wang P. MicroRNA-221 is cardioprotective and anti-fibrotic in a rat model of myocardial infarction. Mol. Ther. Nucleic Acids 17, 185-197 (2019).

27. Chen $Q$, Zhou Y, Richards AM, Wang P. Up-regulation of miRNA-221 inhibits hypoxia/reoxygenation-induced autophagy through the DDIT4/mTORC1 and Tp53inp1/ p62 pathways. Biochem. Biophys. Res. Commun. 474(1), 168-174 (2016).

28. Peng $\mathrm{H}$, Yang $\mathrm{H}$, Xiang $\mathrm{X}$, Li S. MicroRNA-221 participates in cerebral ischemic stroke by modulating endothelial cell function by regulating the PTEN/PI3K/ AKT pathway. Exp. Ther. Med. 19(1), 443-450 (2020). 